\title{
Preferences for ascending and descending hierarchical organization in spatial communication
}

\author{
JODIE M. PLUMERT, THOMAS L. SPALDING, and PENNEY NICHOLS-WHITEHEAD \\ University of Iowa, Iowa City, Iowa
}

\begin{abstract}
People prefer to order spatial information in a hierarchy of decreasing size of spatial unit when giving directions for finding objects and in a hierarchy of increasing size of spatial unit when providing descriptions of object locations (Plumert, Carswell, DeVet, \& Ihrig, 1995). In five experiments, we examined whether people have a preference for ascending or descending organization when the task does not involve conveying spatial information to others. In Experiments 1-3, people learned the locations of objects in a model house and then verified statements describing those locations. People verified statements faster when spatial units were organized in an ascending (i.e., small to large) than in a descending (i.e., large to small) or random order. In Experiment 4, people first performed a sentence verification task and afterward wrote down directions for finding the objects. People again exhibited a preference for ascending organization in the verification task but exhibited a preference for descending organization when giving directions for finding the same objects. Experiment 5 demonstrated that the ascending advantage was not due to the link between the object and small landmark. Discussion focuses on the role of pragmatics and memory retrieval in preferences for ascending versus descending hierarchical organization.
\end{abstract}

Requests for information concerning the whereabouts of missing objects are commonplace in everyday social interaction. Although not all responses to such queries are helpful, asking others for information about missing objects such as checkbooks and car keys can be a highly effective way of locating those objects. The apparent ease with which people give and receive verbal information about location, however, belies a fundamental problem that both speakers and listeners confront when communicating about location. This problem, usually referred to as the linearization problem, arises because there is a mismatch between linguistic and spatial information (Clark, 1985; Levelt, 1982); specifically, speech is linear and space is not. Therefore, when describing how to find something, the speaker must compress two- or threedimensional spatial information into the linear format of speech. Likewise, when comprehending directions for finding something, the listener must interpret linearized spatial information with respect to a two- or threedimensional frame of reference.

One solution to the linearization problem is to convey spatial information in the form of a route. A number of studies have shown that people rely on temporal sequencing of spatial information to describe spatial lay-

We thank Dana Dunisch, Andrea Harmon, Damien Ihrig, Jennifer Kreps, and Azure Welborn for their assistance with data collection and coding. We also thank Lyndon Berglan, Tom Lund, and Jerry Snitselaar for their help with computer programming. Correspondence concerning this article should be addressed to J. M. Plumert, Department of Psychology, 11 SSH East, University of Iowa, Iowa City, IA 52242 (email: jodie-plumert@uiowa.edu). outs (e.g., Levelt, 1982; Linde \& Labov, 1975; UllmerEhrich, 1982). For example, Linde and Labov (1975) found that people described the layout of their apartment by taking the listener on a mental tour through the space. Similarly, Ullmer-Ehrich (1982) found that people sometimes described the layout of the furniture in their dormitory room by taking the listener on a mental gaze tour around the room. That is, each successive furniture item was mentioned relative to the previous one (e.g., "There is a desk to the left of the door. Next to it is a bed. Next to the bed is a chair. Next to the chair is a bookcase."). These studies suggest that conveying spatial information as a sequence of steps along a route is an effective means of linearizing spaces.

Another solution to the linearization problem is to convey spatial information according to a hierarchy of size of spatial unit. For example, one might describe how to find a set of keys by starting with the most general spatial unit and ending with the most specific spatial unit (e.g., "Go upstairs and look in the bedroom on the dresser in the top drawer."). A number of studies have shown that people often use hierarchical organization to structure their descriptions of spatial layouts and object locations (Plumert, Carswell, DeVet, \& Ihrig, 1995; Plumert, Pick, Marks, Kintsch, \& Wegesin, 1994; Shanon, 1984; Taylor \& Tversky, 1992). Plumert et al. (1995), for example, found that people order landmarks and spatial regions according to a hierarchy of size of spatial unit when giving directions for finding objects in a multilevel space (e.g., "Look in the study on the desk in a box next to the telephone."). Taylor and Tversky also found that the order in which adults drew and described spatial layouts learned 
from maps reflected hierarchical structuring of information. Similarly, Shanon found that people tended to describe their dormitory room by referring first to the larger, more stable elements of the room and then to the smaller, moveable things in the room. These studies suggest that conveying spatial information as a sequence of successive steps through a spatial hierarchy is also an effective means of linearizing spaces.

One problem that speakers face when using hierarchical organization to communicate about location is whether to start at the most general or at the most specific level of the hierarchy. Because hierarchies are bi-directional, units of spatial information can be conveyed in either an ascending (i.e., small to large) or a descending (i.e., large to small) order. For example, one could say, "The pen is in the basement in the playroom on the couch under the pillow." or "The pen is under the pillow on the couch in the playroom in the basement." Although both of these organizations clearly specify the location of the object, what seem to be very subtle differences between communication tasks lead to large differences in people's preferences for one organization over the other. In the Plumert et al. (1995) study, people first learned the locations of several objects in a multilevel model house and later either wrote down directions about how to find each object or wrote down descriptions of where each object was located. Thus, the only difference between the two situations was whether the task involved informing the listener about how to find the objects or where the objects were located. Despite the similarities between the two tasks, nearly all hierarchical directions were descending (e.g. "To find the hanger, look on the second floor in the bedroom on the dresser under the hat.") and nearly all hierarchical descriptions were ascending (e.g. "The hanger is under the hat on the dresser in the bedroom on the second floor.").

What are some aspects of these two communication tasks that might lead to differences in how speakers communicate hierarchical information? When the task is to give directions to someone else about how to find something, the goal is to get the listener from his or her present position to the location of the object. Thus, speakers may feel constrained to convey units of spatial information in the order in which they will be encountered by the listener. Movement through the space generally dictates that the listener encounter spatial units (i.e., landmarks and regions) in an order of decreasing size. That is, the listener must reach the floor before the room, and once inside the room, he/she will be likely to notice large landmarks before small landmarks. Thus, when the task involves directing a listener's movements from one point to another, speakers may feel obligated to use descending hierarchical organization. When the task is to describe to someone else where an object is located, the goal is to convey remembered information about the location of the object, but not necessarily to tell the listener how to go about finding the object. For example, if two people are comparing notes on where they store their extra house keys, it is unlikely that they would give each other direc- tions for finding the keys. Without the constraint of directing the listener's movements through space, the speaker may first think of where the object is in relation to an immediate landmark and then try to provide information about where that landmark is in relation to other larger landmarks, and so on. Consequently, hierarchically organized descriptions of object locations may be more likely to start with the most specific link in the hierarchy. Thus, pragmatic concerns may play a critical role in people's preferences for using descending organization to structure directions and ascending organization to structure descriptions.

One question that remains unanswered is whether speakers themselves have a preference for ascending or descending hierarchical organization. In other words, do people have a preference for one type of organization over the other when the task does not involve conveying spatial information to others? As suggested above, the way in which speakers organize spatial information for others may not necessarily reflect the way in which they prefer to organize spatial information for themselves. Other research, in fact, has shown that speakers' preferences for describing spatial relations sometimes override listeners' preferences (e.g., Schober, 1993). For example, Schober (1993) found that speakers tended to use their own perspectives (e.g., "on my left") rather than their listeners' perspectives (e.g., "on your right") when describing locations. Quite likely, speakers found it easier to use their own perspectives than to engage in the transformations necessary to use their listeners' perspectives. In the case of describing object locations, speakers may prefer to think of locations by starting with the most specific level of the hierarchy and then moving to progressively more general levels of the hierarchy. If so, this would suggest that speakers must reorder spatial information from large to small when giving directions for how to find missing objects.

The goal of the present investigation was to test whether people have a preference for ascending or descending hierarchical organization when the task does not involve conveying spatial information to others. We addressed this issue by asking people to verify sentences describing previously learned locations. Sentence verification is ideally suited to address this issue because it should be easier to verify a sentence when the spatial information is presented in an order that matches people's preferences. For example, if people prefer a small to large over a large to small ordering of spatial information, then they should be faster to verify ascending hierarchical sentences as opposed to descending hierarchical sentences. As in Plumert et al. (1995), people first learned the locations of several objects in a multilevel model house. After learning the locations, people were asked to verify sentences describing the locations. Each sentence that people read in the verification task included a reference to the floor, room, large landmark, and small landmark (e.g., "The shoe is on the second floor in the bedroom on the bed under the shirt."). These units of spatial information were organized in an ascending (small to large), a descending 
(large to small), or a random order. In a series of five experiments, we compared people's verification times for sentences organized in ascending versus descending order, descending versus random order, and ascending versus random order. We predicted that people would be faster to verify sentences in which spatial information was ordered from small to large rather than large to small, suggesting a preference for ascending organization over descending organization. We also expected that people would be faster at verifying ascending and descending sentences as opposed to random sentences.

\section{EXPERIMENT 1}

The first experiment was designed to test whether people are faster at verifying ascending or descending hierarchical sentences.

\section{Method}

Participants. Eighteen undergraduates participated for course credit. Two participants were excluded from the experiment and were replaced because more than $25 \%$ of their responses in the sentence verification task were incorrect. All participants were native English speakers.

Apparatus and Materials. A $30 \mathrm{~cm}$ deep $\times 114 \mathrm{~cm}$ wide $\times$ $94 \mathrm{~cm}$ high three-story model house was used as the experimental space. Each of the three levels of the house was subdivided into four rooms. Within each room, two furniture items served as large landmarks. A small landmark was placed on top of each target furniture item. These small landmarks served as hiding places for 24 target objects. Thus, each object location was defined by a small landmark, a large landmark, a room, and a floor. For example, the carrot was on the first floor in the kitchen on the stove in the pot. The Appendix contains the name of each target object and a description of its location. A Macintosh IIfx computer, a 15-in. color monitor, and a standard keyboard were used to control display of sentences in the verification task and to record responses.

Design and Procedure. The session began with a familiarization period in which individual participants were shown the model house. The experimenter informed the participants that they would be asked to learn the locations of several objects in the model house and later recall the locations of those objects. The experimenter named the floors and rooms in the house in a random order and then left the room for $3 \mathrm{~min}$ while the participants studied the model house.

When the experimenter returned, the object placement training began. The experimenter placed the target objects in their proper locations in the model house in a random order. For each location, the experimenter picked up an object and said, "The [object name] goes right here." After placing all of the objects, the experimenter asked the participants to turn around and removed the objects from the model house. The participants were then required to replace all of the objects in their proper locations. The experimenter named and handed each object to the participants in a random order. If the participant made a mistake, the experimenter moved the target object to the correct location. The location-learning procedure was repeated until the participants correctly replaced all 24 target objects twice in succession. The mean number of trials to reach the learning criterion was $2.9(S D=.76)$, ranging from 2 to 5 trials.

The second part of the experiment involved a sentence verification task. The experimenter covered the model house and seated participants at the computer. The experimenter instructed participants that they would be reading sentences about the locations of the objects in the model house and that their task was to decide whether each sentence was true or false. Participants were told to verify each sentence as accurately but as quickly as possible.
Each trial was preceded by a 2-sec intertrial interval in which a blank screen appeared. A single sentence then appeared in the center of the screen. Two rectangular buttons below each sentence were placed to remind participants which key was "true" and which key was "false." The button on the right was labeled "true," and the button on the left was labeled "false." The sentence was displayed until the first keyboard response, at which time the true/false choice and the response latency (to the nearest millisecond) were recorded. Following selection, the button corresponding to the choice made was highlighted momentarily. No feedback was given about the accuracy of the choice. The screen was then cleared for the next intertrial interval to begin.

The verification task started with 11 practice sentences. These sentences were a mixture of true and false general knowledge statements and spatial descriptions unrelated to the model house (e.g., "Northwestern University is in the Midwest in the state of Illinois near Chicago in Evanston" and "Humans have six fingers on each hand."). After completing the practice task, each participant verified 24 sentences corresponding to the locations of the objects in the model house. Each object in the house was represented by one sentence in the verification task. Four units of spatial information (i.e., floor, room, large landmark, and small landmark) made up a complete description of each object location. For half of the sentences, the spatial units were organized in a hierarchy of increasing size of spatial unit (e.g., "The paintbrush is in the bucket on the washer in the laundry room in the basement."), and for the other half they were organized in a hierarchy of decreasing size of spatial unit (e.g., "The paintbrush is in the basement in the laundry room on the washer in the bucket."). For each participant, the computer randomly designated locations as ascending or descending sentences. Half of the ascending and descending sentences were true, and half were false. False sentences were created by randomly selecting a spatial unit (i.e., the floor, room, large landmark, or the small landmark) from a sentence and replacing it with another randomly selected spatial unit from the same level. For example, if the correct floor was the "basement," then the word "basement" in the sentence would be replaced either with "first floor" or "second floor." Thus, a statement was false if any spatial unit in the hierarchy was described incorrectly. This method of constructing false sentences ensured that participants had to consider all spatial units (i.e., floor, room, large landmark, and small landmark) when verifying the true sentences.

Thus, the sentences corresponded to a $2 \times 2$ factorial combination (i.e., true ascending, true descending, false ascending, and false descending), resulting in six sentences of each type. The order of these sentences in the verification procedure was randomized across participants.

\section{Results and Discussion}

The primary question of interest was whether people would find it easier to verify ascending or descending descriptions of the object locations. We addressed this question by comparing mean response times to the true ascending descriptions with mean response times to the true descending descriptions. Response times to false sentences were not analyzed, because participants could often judge that a sentence was false without reading the entire sentence. For example, if the large landmark was incorrect in an ascending hierarchical sentence, participants would only need to read the first part of the sentence in order to decide that it was false. Therefore, responses to true sentences and false sentences were not comparable.

The overall error rate was low, accounting for $5 \%$ of responses on the average. The mean number of errors was $.11(S D=.32)$ for true ascending, $.17(S D=.38)$ for true 
descending, $.50(S D=.51)$ for false ascending, and .44 $(S D=.78)$ for false descending sentences. The mean number of errors for each sentence type (i.e., true ascending, true descending, false ascending, and false descending) was entered into a sentence validity (true vs. false) $\times$ sentence organization (ascending vs. descending) repeated measures analysis of variance (ANOVA). This analysis revealed only a main effect of sentence validity $[F(1,17)=5.67, p<.05]$. People made significantly more errors on false $(M=.14, S D=.35)$ than on true sentences $(M=.47, S D=.65)$. None of the incorrect responses were included in the analyses that follow.

Prior to calculating the means, individual response times that were three or more standard deviations from the mean for each of the two sentence types (i.e., true ascending and true descending) were classified as outliers and removed. The numbers of outliers removed for ascending and descending sentences were one and three, respectively. Mean response times for true ascending and descending sentences were then entered into a one-way repeated measures ANOVA. This analysis revealed that people were significantly faster to verify descriptions of the object locations when units of spatial information were organized in an increasing order of size $(M=5,005 \mathrm{msec}$, $S D=994)$ rather than in a decreasing order of size $(M=$ $5,795 \mathrm{msec}, S D=1,581)[F(1,17)=4.80, p<.05]$. The fact that verification times for ascending descriptions were approximately $15 \%$ faster than those for descending descriptions clearly shows a large preference for ascending organization.

Although the results of the present experiment clearly show that people prefer ascending over descending hierarchical organization, one might expect that people would favor descending hierarchical organization over random organization. That is, given the typically large preference for organized over unorganized materials (e.g., Bower, Clark, Lesgold, \& Winzenz, 1969), people should find it easier to verify sentences when units of spatial information are organized in a descending order of size rather than in a random order of size. Moreover, although there was a large advantage of ascending over descending organization in the present experiment, it is possible that descending hierarchical organization might have an even greater advantage over random organization. Therefore, a second experiment was conducted to test whether people would verify descending hierarchical sentences faster than randomly organized sentences.

\section{EXPERIMENT 2}

\section{Method}

Participants. Eighteen undergraduates participated for course credit. All participants were native English speakers.

Apparatus and Materials. The apparatus and materials were identical to those in the previous experiment.

Design and Procedure. The location-learning procedure was identical to that used in the first experiment. The mean number of trials to reach the learning criterion was $2.6(S D=.50)$, ranging from 2 to 3 trials. The testing procedure was also identical to that used in the first experiment. The only difference was that participants verified sentences that were either descending or random. For each participant, the computer randomly selected which locations to present as descending or random sentences. The following procedure was used to construct the random sentences. For each participant, a random order was randomly chosen for each sentence from a subset of 21 out of the 24 possible permutations of the four spatial units. Only 21 orders were used because the three organizations corresponding to descending, ascending (smallest to largest), and quasi-hierarchical (largest first and then smallest to largest) orderings were excluded from the set of 24 possible orders. ${ }^{1}$ Again, half of the sentences of each type were true and half were false. Thus, the four combinations of sentence type were true descending, true random, false descending, and false random.

\section{Results and Discussion}

We addressed the question of whether people would find it easier to verify descending rather than random spatial descriptions by comparing mean response times to the true descending descriptions with mean response times to the true random descriptions. Again, the overall error rate was low, accounting for $5 \%$ of responses on the average. The mean number of errors was $.28(S D=.58)$ for true descending, $.06(S D=.24)$ for true random, .39 $(S D=.61)$ for false descending, and $.50(S D=.79)$ for false random sentences. The mean number of errors for each sentence type (i.e., true descending, true random, false descending, and false random) was entered into a sentence validity (true vs. false) $\times$ sentence organization (descending vs. random) repeated measures ANOVA. This analysis revealed only a main effect of sentence validity $[F(1,17)=5.74, p<.05]$. People made significantly more errors on false $(M=.44, S D=.70)$ than on true sentences $(M=.17, S D=.45)$. None of the incorrect responses were included in the analyses that follow. Outliers were treated in the same way as in Experiment 1. The numbers of outliers removed for descending and random descriptions were 2 and 2 , respectively.

Mean response times for true descending and random descriptions were entered into a one-way repeated measures ANOVA. Surprisingly, people were not significantly faster to verify descriptions of the object locations when units of spatial information were organized in a decreasing order of size $(M=4,858 \mathrm{msec}, S D=1,188)$ rather than in a random order of size $(M=4,976 \mathrm{msec}, S D=$ 1340) $[F(1,17)=.20$, n.s.]. In fact, response times for descending descriptions were only $2 \%$ faster than response times for random descriptions.

Although we initially thought it obvious that there should be some advantage for organized over unorganized descriptions, the results of this experiment cast doubt on this assumption. However, it may be that only some types of organized descriptions are preferred over randomly organized descriptions. That is, people may prefer ascending hierarchical descriptions over random descriptions, but not descending hierarchical descriptions over random descriptions. A third experiment was conducted, therefore, to test whether verification times for ascending hierarchical sentences were faster than those for random sentences. 


\section{EXPERIMENT 3}

\section{Method}

Participants. Eighteen undergraduates participated for course credit. One participant was excluded from the experiment and replaced because more than $25 \%$ of that participant's responses in the sentence verification task were incorrect. All participants were native English speakers.

Apparatus and Materials. The apparatus and materials were identical to those in the previous experiments.

Design and Procedure. The location-learning procedure was identical to that used in the previous experiments. The mean number of trials to reach the learning criterion was $2.9(S D=.68)$, ranging from 2 to 4 trials. The testing procedure was also identical to that used in the previous experiments. In this experiment, however, participants verified ascending and random sentences. Random sentences were constructed in the same manner as in Experiment 2. For each participant, the computer randomly selected which locations to present as ascending or random sentences. Again, half of the sentences of each type were true and half were false, resulting in four combinations of sentence type corresponding to true ascending, true random, false ascending, and false random.

\section{Results and Discussion}

We addressed the question of whether people found it easier to verify ascending rather than random spatial messages by comparing mean response times to the true ascending descriptions with mean response times to the true random descriptions. Only response times from correctly answered descriptions were analyzed. Again, the overall error rate was low, accounting for $5 \%$ of responses on the average. The mean number of errors was $.28(S D=$ $.58)$ for true ascending, .22 ( $S D=.55)$ for true random, $.17(S D=.51)$ for false ascending, and $.61(S D=.61)$ for false random sentences. The mean number of errors for each sentence type (i.e., true ascending, true random, false ascending, and false random) was entered into a sentence validity (true vs. false) $\times$ sentence organization (ascending vs. random) repeated measures ANOVA. This analysis yielded no significant effects. Outliers were treated in the same way as in the previous experiments. The numbers of outliers removed for ascending and random descriptions were 2 and 2 , respectively.

Mean response times for true ascending and random descriptions were entered into a one-way repeated measures ANOVA. People were significantly faster to verify descriptions of the object locations when units of spatial information were organized in an increasing order of size $(M=4,224 \mathrm{msec}, S D=1,310)$ than in a random order of size $(M=5,189 \mathrm{msec}, S D=1,803)[F(1,17)=$ $13.21, p<.01]$. These results show an overwhelming preference for ascending over random organization. In fact, response times to ascending sentences were $20 \%$ faster than response times to random sentences. This difference is close to the $15 \%$ advantage of ascending over descending hierarchical sentences found in Experiment 1. Clearly, people have a preference for ascending hierarchical organization when verifying descriptions of previously learned locations.

Although the results of Experiment 1 establish a preference for ascending over descending hierarchical orga- nization, a potential methodological problem makes the preference for ascending over random organization less clear. In the present experiment, each participant verified a set of random sentences that were different from one another. That is, the computer selected a new random ordering for each random sentence. Thus, people may have been faster to verify ascending rather than random sentences because they had to comprehend only one ascending ordering but many different random orderings. (Note, however, that this does not explain the lack of an advantage of descending hierarchical descriptions over random descriptions. Rather, using a different random ordering for each sentence should have maximized our chances of finding a significant advantage of descending over random sentences.)

Experiment 4 was conducted to clarify the difference in verification times for ascending and random sentences. In this experiment, we again compared verification times for ascending and random sentences. This time, however, each participant verified a set of random sentences that conformed to a single random ordering. We also thought it worthwhile to replicate the original bias for descending hierarchical organization in direction giving reported by Plumert et al. (1995). Thus, after people performed the sentence verification task, they were asked to write down directions for finding the objects in the model house. This provided a strong test of whether a bias for descending hierarchical organization truly exists in direction giving, because people wrote down directions about locations that they had previously seen described with ascending and with random sentences. That is, although people might be willing to reorganize previously seen random sentences into descending directions, they might be less likely to do so with previously seen ascending sentences. However, if people reorganized ascending descriptions to descending directions, then we would have even more compelling evidence for the descending bias in direction-giving.

\section{EXPERIMENT 4}

\section{Method}

Participants. Twenty undergraduates participated for course credit. Three participants were excluded from the experiment and replaced because more than $25 \%$ of their responses in the sentence verification task were incorrect. All participants were native English speakers.

Apparatus and Materials. The apparatus and materials were identical to those in the previous experiments.

Design and Procedure. The location-learning procedure was identical to that used in the previous experiments. The mean number of trials to reach the learning criterion was $2.7(S D=.88)$, ranging from 2 to 5 trials. After learning the locations, they performed a sentence verification task in which they verified ascending and random sentences. In this experiment, however, the computer randomly chose one random ordering from the 21 available random orderings for each participant. Thus, the random ordering remained consistent across all of the random sentences for each participant.

After the sentence verification task, participants performed a direction-giving task. Following the same procedure as that used in Plumert et al. (1995), the experimenter informed participants that 
"I'm going to hand you several sheets of paper one at a time with the names of the objects written on them. When you get each one, I want you to write down directions for how to find each of those objects so that someone else would know how to look for them on the basis of your directions." They were then handed the pieces of paper one at a time with the sentence "To find the (object name) ..." printed at the top. The participants wrote down directions for finding only the 12 objects that had previously been described with true random and true ascending sentences in the sentence verification task. This procedure was adopted to make the direction-giving task less taxing for the participants. Note, however, that because locations were randomly designated as true and false ascending and random sentences in the sentence verification task, the set of 12 objects used in the direction-giving task varied across participants.

Coding of Directions. Directions for finding the objects were coded for errors, units of spatial information present, ascending versus descending hierarchical organization, and the first spatial unit mentioned. We used the same coding system as in Plumert et al. (1995).

Errors. A message was coded as incorrect when participants gave false information or no information about an object location. The mean numbers of correct messages given for locations corresponding to the ascending and random statements from the sentence verification task were 5.7 and 5.8 out of 6 , respectively $[F(1,19)=.66$, n.s.]. All incorrect messages were excluded from further analyses.

Units of spatial information. Seven types of spatial units were coded in the participants' messages. These units corresponded to seven nested levels of spatial information: (1) floor, (2) floor part, (3) room, (4) room part, (5) large landmark, (6) large landmark part, and (7) small landmark. A reference to a floor was coded as present when participants named the floor or otherwise identif ied a single whole level of the house. A reference to a floor part was coded as present when participants made statements such as "go toward the left side of the basement" or "it's in the front part of the first floor." A reference to a room was coded as present when participants named the room, mentioned its function, or described its appearance. A room part was coded as present when participants made statements such as "look in the corner" or "it's in the right hand side of the living room." A large landmark was coded as present when participants referred to one of the large landmarks. A large landmark part was coded as present when participants made statements about distinct parts of large landmarks such as "Look on the right hand side of the couch." Finally, a small landmark was coded as present when participants referred to one of the small landmarks used as hiding places.

Ascending versus descending hierarchical organization. Directions were coded as ascending when units of spatial information were conveyed in an order of increasing size, and as descending when the units of spatial information were conveyed in an order of decreasing size. Because a minimum of three spatial units is necessary to determine whether or not a direction is hierarchically organized, only directions containing three or more spatial units were included in the analyses of ascending versus descending hierarchical structure. The mean percentages of directions containing three or more spatial units were $91 \%$ and $92 \%$, respectively, for locations corresponding to the ascending and random statements from the sentence verification task.

First spatial unit mentioned. Directions were also coded for whether the largest or the smallest spatial unit was mentioned first. A direction was coded as starting with the largest spatial unit when the largest of the spatial units given in the direction was mentioned first. Likewise, a direction was coded as starting with the smallest spatial unit when the smallest of the spatial units present in the direction was mentioned first.

Reliability. Two coders scored five randomly selected participants' protocols ( $25 \%$ of the sample) so that reliability could be assessed. Reliability for all measures was calculated using exact percent agreement. Intercoder agreement for number of spatial units present, ascending versus descending organization, and the first spatial unit mentioned was high, ranging from $93 \%$ to $96 \%$.

\section{Results and Discussion}

Sentence verification task. As in previous experiments, only response times from correctly answered descriptions were analyzed. Again, the overall error rate was low, accounting for $8 \%$ of responses on the average. The mean number of errors was $.50(S D=.83)$ for true ascending, $.35(S D=.49)$ for true random, $.40(S D=.50)$ for false ascending, and $.60(S D=.94)$ for false random sentences. The mean number of errors for each sentence type (i.e., true ascending, true random, false ascending, and false random) was entered into a sentence validity (true vs. false) $\times$ sentence organization (ascending vs. random) repeated measures ANOVA. This analysis yielded no significant effects. Outliers were treated in the same way as in the previous experiments. The numbers of outliers removed for ascending and random descriptions were 3 and 2, respectively.

As before, people were significantly faster to verify descriptions of the object locations when units of spatial information were organized in an increasing order of size $(M=4,629 \mathrm{msec}, S D=1,200)$ rather than in a random order of size $(M=5,290 \mathrm{msec}, S D=1,621)[F(1,19)=$ $5.47, p<.05]$. Thus, even when the type of random organization remained consistent across sentences, response times for ascending hierarchical statements were $14 \%$ faster than response times for random statements.

Direction-giving task. Given that people again showed a strong preference for ascending hierarchical organization in the sentence verification task, what kind of organization did they use to structure their directions for finding the same objects? First, it is important to note that a large percentage of directions were hierarchically organized $(M=52 \%)$. Moreover, the overall percentage of hierarchical directions about locations corresponding to previously ascending sentences $(M=49 \%)$ and random sentences $(M=55 \%)$ did not differ $[F(1,18)=.94$, n.s. $]$ (1 participant was excluded from this analysis because that participant provided no directions with three or more spatial units).

More importantly, as reported in Plumert et al. (1995), people exhibited a strong bias for descending hierarchical organization in their directions. In the present experiment, $85 \%$ of all hierarchical directions were descending and only $15 \%$ were ascending. Analyses of chance performance $(p=.50)$ revealed that the proportion of descending hierarchical directions was significantly above chance $[t(16)=4.34, p<.001]$ (3 participants were excluded from this analysis because they provided no hierarchical directions). Moreover, virtually all of the ascending hierarchical directions were produced by 2 participants who gave only ascending directions. Without these 2 participants, $97 \%$ of all hierarchical directions that people produced were descending. Interestingly, this preference for descending organization did not differ depending on whether the direction was about a location that previously had been described with an ascending sentence $(M=$ 
$81 \%)$ or with a random sentence $(M=85 \%)[F(1,12)=$ 2.08 , n.s.] (7 participants were excluded from this analysis because they failed to provide hierarchical directions for locations previously described by either random or ascending sentences). Thus, people exhibited a strong bias toward reorganizing spatial units into descending hierarchical directions regardless of whether they had just seen those spatial units organized into ascending or random descriptions.

These results clearly show that when people produced hierarchically organized directions, they almost always ordered units of spatial information from large to small. The fact that about half of all directions were not hierarchically organized, however, raises the question of whether this bias for descending organization extends to nonhierarchically organized directions as well. One way to address this question is to ask whether people were more likely to mention the largest or the smallest spatial unit first in directions containing more than two spatial units. Consistent with the previous results, the largest spatial unit was mentioned first in $71 \%$ of all directions. This preference did not differ depending on whether the direction was about a location that previously had been described with an ascending sentence $(M=67 \%)$ or with a random sentence $(M=75 \%)[F(1,19)=2.88$, n.s.].

\section{Conclusions}

The results from the sentence verification task show that even when the type of random organization remained consistent across the random sentences, people verified ascending sentences faster than random sentences. Thus, these results confirm those of the previous experiments showing that people prefer ascending organization over both descending and random organization. This preference for ascending organization was specific to verifying descriptions of object locations, however. People overwhelmingly preferred descending hierarchical organization to structure their written directions for finding those same objects, even though the direction-giving task immediately followed the sentence verification task.

Together, the results of Experiments 1-4 suggest that people prefer to think of object locations by starting at the smallest spatial unit and proceeding up the hierarchy to the largest spatial unit. In drawing this conclusion, however, we assume that the advantage of ascending organization was not due to a single link in the hierarchy. That is, we assume that the advantage of ascending organization accrued over all links in the hierarchy. However, it is important to note that the target object and small landmark were semantically or thematically related for about half of the locations used in these experiments. ${ }^{2}$ For example, the paintbrush was in the bucket, the ball was under the racket, and the shoe was under the shirt. People may have found it particularly easy to process the link between the target object and the first unit of spatial information (i.e., the small landmark) in ascending sentences. As a result, overall verification times were shorter for ascending than for descending and random sentences. Thus, the link between the target object and the small land- mark may have played a significant role in decreasing verification times for ascending sentences.

One way to address this issue is to determine whether people are faster to verify sentences when the target object is semantically or thematically related to the small landmark. If the semantic/thematic relatedness hypothesis is correct, then people should be faster to verify related than unrelated ascending sentences, but not faster to verify related than unrelated random sentences. Alternatively, people may be faster to verify related as opposed to unrelated ascending sentences and faster to verify related as opposed to unrelated random sentences. This pattern of findings would suggest that a tight association between the target object and small landmark leads to an overall advantage in verification times, but does not account for the ascending advantage in verification times.

Using existing data from Experiment 4, we conducted a set of exploratory analyses to test the semantic/thematic relatedness hypothesis. First, we performed a median split on the target object-small landmark pairs to classify the sentences into two groups: related and unrelated (see the Appendix for related and unrelated target object-small landmark pairs). Using this classification scheme, we then divided the ascending sentences for each subject into related and unrelated ascending sentences, and divided the random sentences for each subject into related and unrelated random sentences. As in previous analyses, incorrect responses and outliers were removed prior to analysis. Related $(M=2.7, S D=.98)$ and unrelated $(M=2.7, S D=1.0)$ sentences were evenly distributed across ascending sentences. Likewise, related $(M=2.6, S D=1.1)$ and unrelated $(M=3.1, S D=.96)$ sentences were relatively evenly distributed across random sentences. In our first analysis, we examined whether response times were faster for related than for unrelated ascending sentences. This analysis revealed that people were faster to verify related $(M=4,154 \mathrm{msec}, S D=$ $1,140)$ as opposed to unrelated $(M=5,086 \mathrm{msec}, S D=$ $1,485)$ ascending sentences $[F(1,15)=5.51, p<.05]$ (4 subjects were excluded from this analysis because they were not presented with at least two related and two unrelated ascending sentences). In our second analysis, we examined whether response times were faster for related than unrelated random sentences. This analysis revealed that people were also faster to verify related $(M=$ $4,768 \mathrm{msec}, \mathrm{S} D=1,452)$ as opposed to unrelated $(M=$ $5,676 \mathrm{msec}, S D=2,054)$ random sentences $[F(1,16)=$ $6.79, p<.05$ ] (3 subjects were excluded from this analysis because they were not presented with at least two related and two unrelated random sentences). Thus, highly associated target objects and small landmarks resulted in faster verification times for both ascending and random sentences. This pattern of findings suggests that semantic/thematic relatedness leads to an overall advantage in verification times but does not account for the ascending advantage in verification times.

Another way to test the semantic/thematic relatedness hypothesis is to eliminate the link between the target object and the small landmark by omitting the target object 
from the sentences describing the locations. If the link between the target object and the small landmark accounts for the ascending advantage, people should not be faster to respond to ascending than to random sentences when the target object is omitted from the sentences. Alternatively, if the link between the target object and the small landmark does not account for the ascending advantage, people should still be faster to respond to ascending than to random sentences.

We conducted a fifth experiment to further test whether the link between the target object and the small landmark would account for the advantage of ascending organization of spatial information. In this experiment, we eliminated the link between the target object and the small landmark by omitting the target object from the sentences describing the locations. Thus, although people learned the connection between the target object and the small landmark during the location-learning procedure, the target-small landmark link was not included in the sentences describing the locations. As in Experiments 3 and 4 , half of the sentences were ascending (e.g., "There is something in the bucket on the washer in the laundry room in the basement.") and half were random (e.g., "There is something in the laundry room on the washer in the bucket in the basement."). Given our previous analyses of the semantic/thematic relatedness hypothesis, we expected that response times would be faster for ascending than for random sentences even when the target object was omitted from the sentences.

\section{EXPERIMENT 5}

\section{Method}

Participants. Eighteen undergraduates participated for course credit. One participant was excluded from the experiment and replaced because more than $25 \%$ of that participant's responses in the sentence verification task were incorrect. All participants were native English speakers.

Apparatus and Materials. The apparatus and materials were identical to those in the previous experiments.

Design and Procedure. The location-learning procedure was identical to that used in the previous experiments. The mean number of trials to reach the learning criterion was $2.4(S D=.50)$, ranging from 2 to 3 trials. After learning the locations, the participants performed a sentence verification task in which they verified ascending and random sentences. As in Experiment 4, the computer randomly chose one random ordering from the 21 available random orderings for each participant. Unlike previous experiments, however, the target object was omitted from the sentences. All sentences began with the phrase "there is something," followed by a reference to the small landmark, large landmark, room, and the floor (e.g., "There is something in the bag on the footstool in the TV room on the second floor."). As in the previous experiments, half of the sentences of each type (i.e., ascending and random) were true and half were false, resulting in four combinations of sentence type corresponding to ascending true, ascending false, random true, and random false.

\section{Results and Discussion}

We addressed the question of whether the link between the target object and the small landmark played a role in the ascending advantage by comparing mean response times to the true ascending sentences with mean response times to the true random sentences. Only response times from correctly answered sentences were analyzed. Again, the overall error rate was low, accounting for $8 \%$ of responses on the average. The mean number of errors was $.17(S D=.38)$ for true ascending, .17 $(S D=.38)$ for true random, $.72(S D=1.07)$ for false ascending, and $.94(S D=.87)$ for false random sentences. The mean number of errors for each sentence type (i.e., true ascending, true random, false ascending, and false random) was entered into a sentence validity (true vs. false) $X$ sentence organization (ascending vs. random) repeated measures ANOVA. This analysis revealed only a main effect of sentence validity $[F(1,17)=9.38, p<.01]$. People made significantly more errors on false $(M=.83$, $S D=.97)$ than on true sentences $(M=.17, S D=.38)$. Outliers were treated in the same way as in the previous experiments. The numbers of outliers removed for true ascending and true random sentences were 1 and 2 , respectively.

As before, people were significantly faster to verify descriptions of the object locations when units of spatial information were organized in an increasing order of size ( $M=5,392 \mathrm{msec}, S D=1,410 \mathrm{msec})$ than in a random order of size $(M=6,368 \mathrm{msec}, S D=1,958)[F(1,17)=$ $5.71, p<.05]$. These results show clearly that the link between the target object and the small landmark does not account for the advantage of ascending sentences over descending and random sentences. Even without the target object in the sentences, people again exhibited a strong preference for ascending over random organization. As in Experiment 4, response times for ascending sentences were $15 \%$ faster than response times for random sentences. However, omitting the target object from the sentences did have an impact on overall response times. On the average, overall response times were $15 \%$ slower in Experiment 5 than in Experiment 4. A direct statistical comparison of response times in Experiments 4 and 5 confirmed this observation. Response times for ascending and random sentences were entered into an experiment $(4$ vs. 5$) \times$ sentence type (ascending vs. random) repeated measures ANOVA with the first factor as a between-subjects variable and the second as a withinsubjects variable. This analysis yielded a significant effect of experiment $[F(1,36)=4.26, p<.05]$. The mean response time was $4,960 \mathrm{msec}(S D=1,447)$ for Experiment 4 and 5,880 $\mathrm{msec}(S D=1,753)$ for Experiment 5, suggesting that the target object served as an important cue for retrieving spatial information from memory. It is important to note, however, that omitting the target object did not differentially affect ascending and random sentences. Response times for both types of sentences were slower in Experiment 5 than in Experiment 4. This further supports the conclusion that the link between the target object and the small landmark does not account for the advantage of ascending sentences.

As in Experiment 4, we again carried out a set of exploratory analyses to test whether people were faster to respond to related than to unrelated ascending sentences 
and were faster to respond to related than to unrelated random sentences. Although the participants did not see the target objects in the sentences, they presumably learned the link between the target objects and small landmarks during the location-learning procedure. If the experience of learning the link between the target object and small landmark led to the advantage for related sentences, people should again be faster to verify related than unrelated sentences. Alternatively, if the target object was necessary to cue the link between the target object and the small landmark, people should not be faster to verify related than unrelated sentences.

Using the scheme devised in Experiment 4, we divided the ascending sentences for each subject into related and unrelated ascending sentences, and divided the random sentences for each subject into related and unrelated random sentences. As in previous analyses, incorrect responses and outliers were removed prior to analysis. Related $(M=2.7, S D=1.2)$ and unrelated $(M=3.1$, $S D=1.2)$ sentences were relatively evenly distributed across ascending sentences. Likewise, related $(M=2.8$, $S D=1.2)$ and unrelated $(M=2.8, S D=1.1)$ sentences were relatively evenly distributed across random sentences. We first examined whether response times were faster for related than unrelated ascending sentences. This analysis revealed that people were not faster to verify related $(M=5,436 \mathrm{msec}, S D=1,399)$ as opposed to unrelated $(M=5,724 \mathrm{msec}, S D=2,434)$ ascending sentences $[F(1,13)=.18$, n.s. $]$ (4 subjects were excluded from this analysis because they were not presented with at least two related and two unrelated ascending sentences). We then examined whether response times were faster for related than for for unrelated random sentences. This analysis revealed that people were not faster to verify related $(M=6,841 \mathrm{msec}, S D=2,713)$ as opposed to unrelated $(M=6,244 \mathrm{msec}, S D=2,387)$ random sentences $[F(1,14)=.50$, n.s. $]$ ( 3 subjects were excluded from this analysis because they were not presented with at least two related and two unrelated random sentences). Thus, highly associated target objects and small landmarks did not result in faster verification times for either ascending or random sentences. This pattern of findings suggests that semantic/thematic relatedness leads to an overall advantage in verification times only when the target object is present to cue the target object-small landmark link.

\section{GENERAL DISCUSSION}

Across the five experiments, a clear picture emerges. Ascending hierarchical sentences are more easily verified than are randomly organized or descending hierarchical sentences. Moreover, the magnitude of the ascending advantage was highly consistent across experiments. That is, response times for ascending sentences were approximately $15 \%$ faster than response times for both descending sentences and random sentences, and response times to descending and random sentences were virtually identical. Consistent with the results of Plumert et al.
(1995), we also found that when people produced directions for finding a given object, they gave mainly descending hierarchical directions, even if they had just seen the same information organized as an ascending hierarchical description. In addition, the results of Experiment 5 clearly show that the ascending advantage was not due to the link between the target object and the small landmark. Rather, it appears that the advantage for ascending organization accrued over all links in the hierarchy.

Why might this bias for ascending organization exist? There are at least two plausible explanations for this phenomenon. One of these concerns how spatial information might be represented in memory (see also McNamara, 1986). Consider the location of a key to a laboratory room. One might represent the location of the key as in a drawer in a desk in an office on the second floor of the psychology building. In this hierarchically organized representation, each unit of spatial information is represented in relation to the next largest unit of spatial information. Thus, the key is represented in relation to the drawer, the drawer is represented in relation to the desk, the desk is represented in relation to the office, and the office is represented in relation to the second floor of the psychology building. Given this organization, priming of information in memory is likely to proceed in an ascending direction, particularly when the starting point is the object itself. Thus, the key should cue the drawer, the drawer should cue the desk, the desk should cue the office, and so on. The results of Experiment 5 suggest that the object serves as an important cue for accessing memory for location. That is, overall verification times were faster when the target object was included in the sentence. However, it is also important to point out that even without the target object in the sentences, people were faster to verify ascending than random sentences. This suggests that the object itself is not necessary to set the ascending cuing advantage into motion. Thus, the direction of movement through the hierarchy rather than the point of entry into the hierarchy appears to be critical for setting the ascending advantage into motion.

Another explanation of the ascending advantage concerns an asymmetry between individual levels of the hierarchy (Collins \& Quillian, 1969). In fact, if one assumes a strict hierarchical model of spatial memory (e.g., Hirtle \& Jonides, 1985; McNamara, 1986; Stevens \& Coupe, 1978), then priming of spatial information should occur more quickly in an order of ascending, rather than descending, size of spatial unit. That is, there are fewer possible locations to consider at each level of the hierarchy when retrieving spatial information in an ascending rather than in a descending order. Consider the relation between a room and a floor. When one is moving down the hierarchy, there are a number of rooms on the floor. The other rooms linked to the floor are likely to compete with the target room. That is, as the floor is activated, all the rooms connected to the floor are activated. Therefore, the target room must be differentiated from the other activated rooms. On the other hand, if one is moving up the hierarchy, such competition is unlikely to occur because the 
room is only on one floor, the target floor. Such asymmetries could lead to a preference for the ascending organization in verifying sentences describing object locations. General principles of memory retrieval have also been used to explain other spatial biases such as why distance estimates between reference points and nonreference points are asymmetrical (McNamara \& Diwadkar, 1997; Newcombe, Huttenlocher, Sandberg, Lie, \& Johnson, 1999; Sadalla, Burroughs, \& Staplin, 1980).

The retrieval-based explanations outlined above are not mutually exclusive, and there may well be other advantages for the ascending organization. Moreover, although the sentence verification task used here was well suited to address differences in people's preferences for ascending as opposed to descending hierarchical organization, this task does push people to represent and retrieve information about location in a linear fashion. Clearly, there are other ways in which people might think about spatial information. Further research is needed so that we may better understand how the task influences the processes underlying the retrieval of spatial information from memory.

Another possible explanation of the ascending advantage is that there is less referential ambiguity in ascending than in descending spatial descriptions. Consider the following descriptions: (1) "The calculator is under a notebook on the desk in the office"; and (2) "The calculator is in the office on the desk under a notebook." From an objective standpoint, there is more referential ambiguity between the spatial units in the second description than in the first description. For example, one might interpret the phrase "in the office on the desk" to mean that the office was on the desk. Such referential ambiguity could lead to parsing problems when trying to verify descending descriptions. This explanation for the ascending advantage seems unlikely, however. That is, if descending organization is really more referentially ambiguous than ascending organization, then speakers should not rely so heavily on descending hierarchical organization when giving directions to others for finding objects.

One implication of the idea that people find it easier to retrieve spatial information in an order of small to large is that they reorder this information from large to small when giving directions to others for finding objects. This conclusion is supported by the fact that the participants in Experiment 4 strongly preferred ascending organization in the initial verification task and yet preferred descending organization in the subsequent directiongiving task. The idea that people reorder spatial information when giving directions for finding objects may provide an explanation of one aspect of the present results that was somewhat puzzling. Given the usually large advantage for organized over unorganized materials (e.g., Bower et al., 1969), it is surprising that we did not find a reliable difference between the descending hierarchical and the randomly organized descriptions. That is, although some organizations may be better than others, almost any organization is usually better than no organi- zation. If the units of spatial information in a description have to be reordered to match the order in which those units are retrieved from memory (or vice versa), however, then descending descriptions would likely offer no particular advantage over random descriptions. For example, assume that the spatial units of the description must be reorganized so that they are in an ascending order to be matched against the representation of the object location. Each sentence includes four spatial units. Obviously, for an ascending sentence, none of the spatial units would need to be reordered. For a descending sentence, however, all four units would need to be reordered (e.g., basementden-desk-phone to phone-desk-den-basement). Random sentences, on the other hand, would sometimes need all four units reordered (e.g., den-basement-phone-desk to phone-desk-den-basement), but would often need only two or three spatial units reordered (e.g., phone-dendesk-basement to phone-desk-den-basement). This difference in the degree of mismatch from the ascending order might offset any advantage that descending sentences might accrue simply from being organized.

In conclusion, it is clear that people are able to use hierarchical spatial representations quite flexibly in the service of specific tasks. In particular, people prefer descending organization when conveying spatial information in directions for finding objects, but they prefer ascending organization when conveying spatial information in descriptions of object locations. Nonetheless, the present results suggest that people find it easier to retrieve such spatial information from memory in an ascending order. Thus, it appears that people's preference for the ascending or descending hierarchical organization when communicating spatial information to others is dependent on specific task constraints. Investigation of the requirements of specific communication tasks is needed in order to develop a full understanding of how such tasks drive preferences for particular organizations of spatial information.

\section{REFERENCES}

Bower, G. H., Clark, M. C., Lesgold, A. M., \& Winzenz, D. (1969). Hierarchical retrieval schemes in recall of categorical word lists. Journal of Verbal Learning \& Verbal Behavior, 8, 323-343.

Clark, H. H. (1985). Language use and language users. In G. Lindzey \& E. Aronson (Eds.), The handbook of social psychology (3rd ed., Vol. 2, pp. 179-231). New York: Harper \& Row.

Collins, A. M., \& Quillian, M. R. (1969). Retrieval time from semantic memory. Journal of Verbal Learning \& Verbal Behavior, 8, 240-247.

Hirtle, S. C., \& Jonides, J. (1985). Evidence of hierarchies in cognitive maps. Memory \& Cognition, 13, 208-217.

LeVelt, W. J. M. (1982). Linearization in describing spatial networks. In S. Peters \& E. Saarinen (Eds.), Processes, beliefs, and questions: Essays on formal semantics of natural language and natural language processing (pp. 199-220). Boston: D. Reidel.

Linde, C., \& LABov, W. (1975). Spatial networks as a site for the study of language and thought. Language, 51, 924-939.

McNamara, T. P. (1986). Mental representations of spatial relations. Cognitive Psychology, 18, 87-121.

McNamara, T. P., \& DiwadKar, V. A. (1997). Symmetry and asymmetry of human spatial memory. Cognitive Psychology, 34, 160-190. 
Newcombe, N., Huttenlocher, J., Sandberg, E., Lie, E., \& Johnson, S. (1999). What do misestimations and asymmetries in spatial judgment indicate about spatial representation? Journal of Experimental Psychology: Learning, Memory, \& Cognition, 25, 986996.

Plumert, J. M., Carswell, C., DeVet, K., \& Ihrig, D. (1995). The content and organization of communication about object locations. Journal of Memory \& Language, 34, 477-498.

Plumert, J. M., Pick, H. L., Jr., Marks, R. A., Kintsch, A. S., \& WeGESIN, D. (1994). Locating objects and communicating about locations: Organizational differences in children's searching and directiongiving. Developmental Psychology, 30, 443-453.

Sadalla, E. K., Burroughs, W. J., \& Staplin, L. J. (1980). Reference points in spatial cognition. Journal of Experimental Psychology: Human Learning \& Memory, 6, 516-528.

SCHOBER, M. F. (1993). Spatial perspective-taking in conversation. Cognition, 47, 1-24.
Shanon, B. (1984). Room descriptions. Discourse Processes, 7, 225-255.

Stevens, A., \& Coupe, P. (1978). Distortions in judged spatial relations. Cognitive Psychology, 10, 422-437.

TAYlor, H. A., \& Tversky, B. (1992). Descriptions and depictions of environments. Memory \& Cognition, 20, 483-496.

UlLmer-Ehrich, V. (1982). The structure of living space descriptions. In R. J. Jarvella \& W. Klein (Eds.), Speech, place, and action: Studies of deixis and gesture (pp. 219-249). Chichester, U.K.: Wiley.

\section{NOTES}

1. Quasi-hierarchical organization was not used because Plumert et al. (1995) found that people often use quasi-hierarchical organization in their production of spatial messages. Thus, including quasi-hierarchical organization as a random organization would be misleading.

2. We thank our reviewers, David Uttal and Steffen Werner, for suggesting the semantic/thematic relatedness hypothesis.

\section{APPENDIX \\ Objects and Locations}

\begin{tabular}{|c|c|}
\hline Object & Location \\
\hline $\begin{array}{l}\text { 1. Paintbrusha } \\
\text { 2. Detergent } \\
\text { 3. Balla } \\
\text { 4. Glove } \\
\text { 5. Calendar } \\
\text { 6. Letter } \\
\text { 7. Light bulb } \\
\text { 8. Hammerb } \\
\text { 9. Carrota } \\
\text { 10. Knife } \\
\text { 11. Glass } \\
\text { 12. Plate } \\
\text { 13. Skates } \\
\text { 14. Book } \\
\text { 15. Bottle } \\
\text { 16. Cup } \\
\text { 17. Candy barb } \\
\text { 18. Pop can } \\
\text { 19. Toilet paper } \\
\text { 20. Soap } \\
\text { 21. Yarn } \\
\text { 22. Ruler } \\
\text { 23. Hangerb } \\
\text { 24. Shoe }\end{array}$ & $\begin{array}{l}\text { in the bucket on the washer in the laundry room in the basement } \\
\text { under the dustpan on the sink in the laundry room in the basement } \\
\text { under the racket on the pool table in the playroom in the basement } \\
\text { under the helmet on the rocking horse in the playroom in the basement } \\
\text { under the plant on the filing cabinet in the office in the basement } \\
\text { in the briefcase on the desk in the office in the basement } \\
\text { under the shovel on the sawhorse in the workshop in the basement } \\
\text { under the sled on the workbench in the workshop in the basement } \\
\text { in the pot on the stove in the kitchen on the first floor } \\
\text { under the toaster on the refrigerator in the kitchen on the first floor } \\
\text { in the bowl on the china cabinet in the dining room on the first floor } \\
\text { under the tray on the table in the dining room on the first floor } \\
\text { in the box on the bench in the entryway on the first floor } \\
\text { under the present on the bookshelf in the entryway on the first floor } \\
\text { in the vase on the piano in the living room on the first floor } \\
\text { under the blanket on the couch in the living room on the first floor } \\
\text { under the Kleenex on the TV in the TV room on the second floor } \\
\text { in the bag on the footstool in the TV room on the second floor } \\
\text { in the trashcan on the toilet in the bathroom on the second floor. } \\
\text { under the towel on the bathtub in the bathroom on the second floor } \\
\text { in the basket on the sewing machine in the sewing room on the second floor } \\
\text { under the pillow on the chair in the sewing room on the second floor } \\
\text { under the hat on the dresser in the bedroom on the second floor } \\
\text { under the shirt on the bed in the bedroom on the second floor }\end{array}$ \\
\hline
\end{tabular}

aRelated target object-small landmark pair. ${ }^{\mathrm{b}}$ Unrelated target object-small landmark pair. 